\title{
A Near Infrared Radiation Model in a Biological Tissue
}

\author{
H. Trabelsi, M. Gantri and E. Sediki. \\ Unité de Rayonnement thermique - Département de Physique. Faculté des Sciences de Tunis, 2092EL ManarI1, \\ Tunisia.
}

\section{Abstract.}

In this paper, we present a performed solution of a near infrared radiation through a biological tissue . This solution is obtained, in the case of variable refractive index, by using a finite volume discrete ordinate method. In medical imaging, this could be an optical tomography forward model. We consider a very thin rectangular biological tissue-like domain which is submitted to an infrared light source. The source is assumed to be monochromatic, isotropic and time-independent. Transmitted fluxes, in the detector points at the boundaries, are calculated. The proposed model is tested in some experimental cases reported in the literature. Also it is used to study infrared radiation in a multilayer heterogeneous medium. We simulate radiation in skin, fat and muscle.

In fact, optical tomography can be used as a diagnostic volumetric medical imaging modality. It uses visible and near-infrared radiation essentially [1]. One way to model propagation of near-infrared light in this kind of media is diffusion equation. It is an asymptotic behavior of the photon transport equation in very high scattering biological tissue. However, low scattering and highly absorbing regions could not be well represented through this modeling way [2]. For a general biological tissue, it would be more convenient to use a forward model based on radiative transfer equation (RTE). So, providing solutions to the RTE is a crucial research task in the fields of biological tissue optics. In this work, infrared light $(800 \mathrm{~nm}-1100 \mathrm{~nm})$ propagation in a human biological tissue is modeled using a spatially varying refractive index radiative transfer equation as [3]:

$$
\begin{aligned}
\frac{n(\boldsymbol{r})}{c} \frac{\partial \Psi(\boldsymbol{r}, \boldsymbol{\Omega}, t)}{\partial t}+ & \Omega \cdot \boldsymbol{\nabla} \Psi(\boldsymbol{r}, \boldsymbol{\Omega}, t)+\left(\mu_{s}(\boldsymbol{r})+\mu_{a}(\boldsymbol{r})\right) \Psi(\boldsymbol{r}, \boldsymbol{\Omega}, t)+\frac{1}{n(\boldsymbol{r})} \nabla n(\boldsymbol{r}) \cdot \nabla_{\boldsymbol{\Omega}} \Psi(\boldsymbol{r}, \boldsymbol{\Omega}, t) \\
& -\frac{2}{n(\boldsymbol{r})}(\boldsymbol{\Omega} \cdot \boldsymbol{\nabla} n(\boldsymbol{r})) \Psi(\boldsymbol{r}, \boldsymbol{\Omega}, t) \\
& =S(\boldsymbol{r}, \boldsymbol{\Omega}, t)+\mu_{s}(\boldsymbol{r}) \int_{0}^{2 \pi} p(\boldsymbol{\Omega}, \boldsymbol{\Omega}) \Psi(\boldsymbol{r}, \boldsymbol{\Omega}, t) d \boldsymbol{\Omega}
\end{aligned}
$$

In this equation, $\Psi(\boldsymbol{r}, \boldsymbol{\Omega}, t)$ is the directional energetic radiance at the spatial position $\mathbf{r}$ and $n(\boldsymbol{r})$ is the refractive index distribution. Equation (1) takes into account the fact that the light rays are not straight lines but curves. It involves terms that illustrate the expansion or the contraction of the cross section of the tube of light rays in the medium [4].

The radiance in the boundary is the sum of the external source contribution and the partly-reflected radiance due to the refractive index mismatch at the boundary. This gives:

$\Psi\left(\boldsymbol{r}_{b}, \boldsymbol{\Omega}, t\right)=S\left(\boldsymbol{r}_{b}, \boldsymbol{\Omega}, t\right)+R \Psi\left(\boldsymbol{r}_{b}, \boldsymbol{\Omega}_{\mathrm{ref}}, t\right), \quad \boldsymbol{n}_{b} \cdot \boldsymbol{\Omega}<0$ and $\boldsymbol{\Omega}_{\mathrm{ref}} \cdot \boldsymbol{n}_{b}=-\boldsymbol{\Omega} \cdot \boldsymbol{n}_{b}$ where $r_{b}$ is a position-vector on the boundary and $n_{b}$ is a unit outer normal vector. The reflectivity $\mathrm{R}$ can be calculated for each direction using Fresnel's relationships.

In the computational implementation, we use a rectangular domain which is divided into a set of IxJ elementary uniform volumes $\Delta \mathrm{V}$ with a uniform unitary depth. This yields a set of $\mathrm{I}^{*} \mathrm{~J}$ rectangular cells. The angular discretization is obtained through a discrete ordinate method. This yields a set of $\mathrm{M}$ angular discrete direction unit vector ordinates $(\xi \mathrm{m}, \eta \mathrm{m})$. an orientation depending on the incident radiation is given for each cell, as shown in fig. 1. Calculus is carried out by integration of equation (1) over an elementary volume $\Delta \mathrm{V}$ for each discrete direction. Numerical results are obtained for a variety of tissue-like media. Infrared radiative properties are taken from literature [5]. 


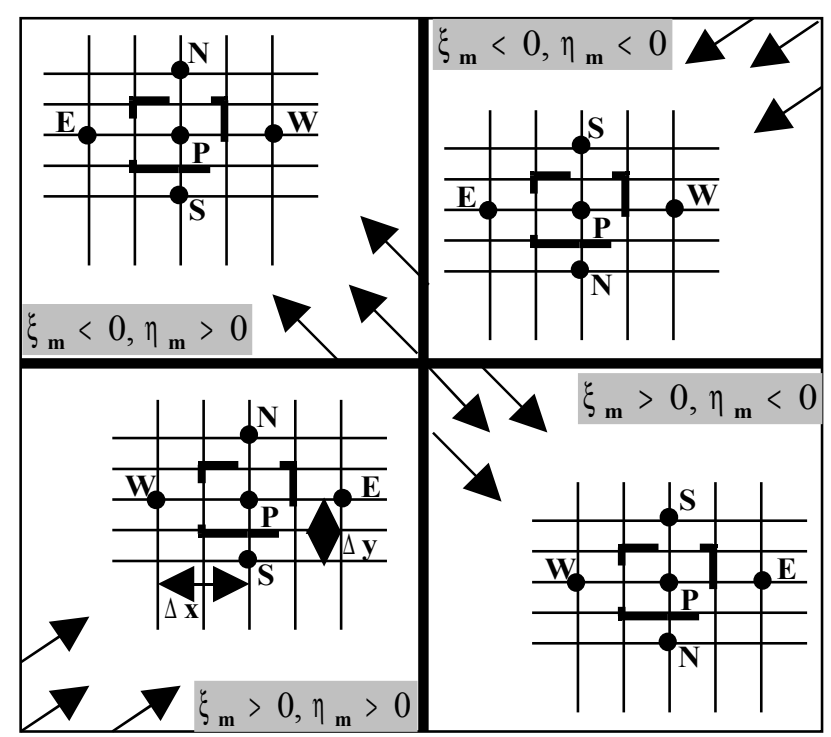

Fig. 1. Spatial finite volume discretization and orientation of cells in the calculus domain.

Keywords: Infrared radiation, Optical tomography, variable refractive index, Biological tissue.

\section{REFERENCES}

1. Arridge, S.R. Optical tomography in medical imaging. Inverse Problems, 15 (1999) 41-93.

2. Klose, A. D., Hielscher, A. H., Optical tomography using the time- independent equation of radiative transfer. Part I: Forward model, JQSRT, 72 (2002) 691-713.

3. T. Khan and A. Thomas. Comparison of PN or spherical harmonics approximation for scattering media with spatially varying and spatially constant refractive indices. Optics Communications, 255 (2005) 130-166.

4. H. Ferwerda. The radiative transfer equation for scattering media with spatially varying refractive index. J. Opt. A: Pure Appl. Opt., 1(1999) L1-L2.

5. Cheong, W.F., Prahl, S.A., and Welch, A.J., A Review of the Optical Properties of Biological Tissues. IEEE J. Quantum Electronics, 26(1990) 2166-2185. 